\title{
Optimal Transmission Thresholds for QoS Guarantees in WSNs
}

\author{
EDGAR ROMO-MONTIEL, Computer Research Center, Instituto Politécnico
}

Nacional, Mexico

MARIO EDUARDO RIVERO-ÁNGELES, Computer Research Center, Instituto Politécnico Nacional, Mexico

HERÓN MOLINA-LOZANO, Computer Research Center, Instituto Politécnico

Nacional, Mexico

GERARDO RUBINO, INRIA Rennes - Bretagne Atlantique, Campus universitaire de Beaulieu,, France

RICARDO MENCHACA-MÉNDEZ, Computer Research Center, Instituto

Politécnico Nacional, Mexico

ROLANDO MENCHACA-MÉNDEZ, Computer Research Center, Instituto

Politécnico Nacional, Mexico

In Wireless Sensor Networks (WSNs), each node typically transmits several control and data packets in a contention fashion to the sink. In this work, we mathematically analyze and study three unscheduled transmission schemes for control packets in a cluster-based architecture named Fixed Scheme (FS), Adaptive by Estimation Scheme (AES) and Adaptive by Gamma Scheme (AGS), in order to offer QoS guarantees in terms of system lifetime (related to energy consumption) and reporting delay (related to cluster formation delay). In the literature, different adaptive schemes have been proposed, and also there is research about the appropriate value selection of the transmission probability for the cluster formation. However, it largely overlooked the minimum and maximum values for the transmission probability that entails the best performance. Based on the numerical results, we show that the threshold values are just as

Authors' addresses: Edgar Romo-Montiel, Computer Research Center, Instituto Politécnico Nacional, Mexico City, Mexico, eromom0900@alumno.ipn.mx; Mario Eduardo Rivero-Ángeles, Computer Research Center, Instituto Politécnico Nacional, Mexico City, Mexico, mriveroa@ipn.mx; Herón Molina-Lozano, Computer Research Center, Instituto Politécnico Nacional, Mexico City, Mexico, hmolina@cic.ipn.mx; Gerardo Rubino, INRIA Rennes - Bretagne Atlantique, Campus universitaire de Beaulieu, Rennes Cedex, France, Gerardo.Rubino@inria.fr; Ricardo Menchaca-Méndez, Computer Research Center, Instituto Politécnico Nacional, Mexico City, Mexico, ric@cic.ipn.mx; Rolando Menchaca-Méndez, Computer Research Center, Instituto Politécnico Nacional, Mexico City, Mexico, rmen@cic.ipn.mx. 
important in the system design as the actual value of the transmission probability in adaptive schemes (AES and AGS), to achieve QoS guarantees.

Additional Key Words and Phrases: WSNs, Transmission, Model, QoS

\section{INTRODUCTION}

Wireless Sensor Networks (WSN) are a set of tiny and autonomous devices called nodes enabled to sense environmental conditions in the area where they are deployed. Additionally, WSNs also have at least one base station or sink node which collects the information gathered by each node [9].

In its most basic form, sensor nodes are devices composed by a transceiver, a sensor, a battery and a processing unit. Meanwhile, the sink node can be a specific device with higher processing, memory and energy capabilities (either with high capacity batteries or with no constraints at all when connected to an electric outlet or by renewable unlimited sources like solar [12] or energy harvesting techniques [14]) or, sometimes, it can be a regular node with higher energy capabilities.

Since the energy source of normal nodes is a standard battery, it is relevant to reduce energy consumption as much as possible in order to prolong the lifetime of the network [4], [6]. Indeed, system lifetime is a principal QoS parameter in the design of WSNs, which is directly related to the energy consumption in the system.

On the other hand, cluster-based architectures have been proven to reduce energy consumption by grouping neighbor nodes into a common cluster [3], [2], [10], and [16]. In such schemes, a node acts as Cluster Head $(\mathrm{CH})$ and the rest of the nodes in the cluster become Cluster Members (CMs). Hence, CMs perform short-range transmissions to the $\mathrm{CH}$ instead of long-range or multi-hop transmissions to the sink. That means that the number of long-range transmissions reduces to only one per cluster.

We focus our study in clustered WSNs, where nodes have to transmit a short control packet at the beginning of the cluster formation phase using a contention-based mechanism, such as the NP/CSMA (Non-Persistent Carrier Sense Multiple Access) [1] while preferring a collision-free scheme to transmit their data once that forming the clusters, such as a TDMA (Time Division Multiple Access) [7] based scheme. The cluster formation phase is highly energy consuming due to the fact that packets are prone to collide with other control packets or in case that no node transmits, the channel can be sub-utilized. Both events compromise the QoS guarantees of the system since collisions cause that nodes must retransmit and sub-utilized channel affects the delay of packets. Later, in the steady phase, nodes have a specific time assigned to transmitting data in a collision-free scheme, such that they can reduce their transmission coverage to reach its $\mathrm{CH}$, and by multi-hop transmissions of short-range, that consumes less energy, reach the sink. 
Additionally, these processes of cluster formation phase and steady phase are performed periodically at certain time in order to distribute the energy load among the nodes in the network, this period is typically known as round.

As such, by reducing energy consumption in the cluster formation phase and reducing cluster formation delay, the WSN can offer QoS guarantees in terms of system lifetime and reporting delay. Note that during the cluster formation phase of each round, nodes cannot convey their data to the sink, which entails that nodes have to store the sensed data in a queue and wait until they are able to transmit such data in the steady phase, it directly affects the reporting delay from nodes to sink.

Building on this, the use of adaptive mechanisms to adjust the transmission probability in the cluster formation phase are widely used to reduce both energy consumption and reporting delay [13], [8] and [5]. Such schemes are designed based on the fact that at the beginning of the cluster formation phase, there are many nodes attempting a transmission, which leads to select a low transmission probability to avoid collisions. Conversely, at the end of this phase, a high transmission probability is preferred since there are just a few nodes left attempting to transmit their control packet, thus reducing empty slots and cluster formation delay.

In this regard, adaptive schemes convey the use of a maximum transmission probability (the value used at the beginning of the cluster formation phase) and a minimum transmission probability (at the end of such phase). Such schemes have been proposed and study in previous works [15]. However, to the best of our knowledge, the selection and performance evaluation of threshold values for the transmission probability has not been studied in detail. In this work, we focus on the analysis of the impact of such threshold values for QoS guarantees in WSNs. To this end, we mathematically study the average energy consumption and average cluster formation delay and obtain numerical results to provide clear guidelines for the selection of such values. We consider two adaptive schemes, namely, Adaptive by Estimation Scheme (AES) and Adaptive by Gamma Scheme (AGS) and prove that threshold transmission probability values have to be chosen carefully to provide QoS guarantees. Additionally, we consider a Fixed Transmission scheme (FS) for comparative purposes.

The rest of the paper is organized as follows: Section 2 presents the system model as well as the main assumptions in this work. Then, in Section 3, the proposed mechanisms are explained in detail. Section 4 describes the threshold selection for QoS guarantees for these schemes. We finish this paper presenting relevant numerical results and conclusions.

\section{SYSTEM MODEL}

In this work, we assume that nodes are located uniformly in the network area and transmit with enough power to be detected by each one of the network members independently of its position. 
4 - E. Romo-Montiel et al.

Table 1. Parameters

\begin{tabular}{|c|c|}
\hline Parameter & Value \\
\hline Nodes in the network & $\mathrm{N}=5,10,20,50,100$ \\
\hline Network size & 100 sqr meters \\
\hline Sample size & $\begin{array}{l}100000 \text { networks of } 100 \\
\text { nodes }\end{array}$ \\
\hline Time of transmission & 1 unit (1 time slot) \\
\hline $\begin{array}{l}\text { Energy consumption } \\
\text { for Tx }\end{array}$ & 1 unit(50nJ/bit) \\
\hline $\begin{array}{l}\text { Energy consumption } \\
\text { for Rx }\end{array}$ & 1 unit(50nJ/bit) \\
\hline
\end{tabular}

Once the nodes positioned, the number of nodes and their location do not change in the time. We focus our study in the cluster formation process, similar to the LEACH protocol [11] under a CSMA/NP scheme as the MAC protocol. In order to study the performance of the schemes proposed in this work, we used the parameters shown in Table 1.

As LEACH considers rounds with cluster formation and steady-state phases, we assume that the cluster formation process must perform periodically to distribute energy inside the network, since CHs drain energy faster than CMs. Thus, rounds prevent early death of a set of nodes (CHs).

We are using the First Order Radio Model described in [11] as the energy model with the consumptions shown in Table 1. We consider an error-free and slotted in the time channel, in each slot, only one node can successfully transmit its control packet.

\section{ADAPTIVE TRANSMISSION SCHEMES}

In this section, we describe the three mechanisms proposed to reduce the energy consumption in the sending of control packets in a cluster-based WSNs similar to LEACH (Low Energy Adaptive Clustering Hierarchy) [11]. We consider a slotted channel. As such, the transmission of control packets is limited by a transmission probability at the beginning of each slot.

Since nodes transmit in a contention scheme, collisions and idle slots are susceptible to occur. This events are undesirable because, in the case of collision, it demands that nodes have to retransmit their packets consuming extra energy and increasing the delay. While idle slots, due to the lack of transmissions in a given slot, entails extra delay and also extra energy consumption since nodes have to continuously listen to the channel during 
the complete cluster formation phase to know when this phase concludes and thus when the steady state phase is beginning for the data transmission procedure.

A node that has not successfully transmitted its control packet before transmits with transmission probability $\tau$ at the beginning of a time slot. However, if the amount of nodes attempting to transmit is high, the value of $\tau$ needs to be low, conversely, if the amount of nodes attempting to transmit is low, the value of $\tau$ could take higher values in order to give the channel access to one node with information waiting to be sent and avoid idle slots. Now, we describe the adaptive transmission schemes in detail.

\subsection{Fixed Scheme (FS)}

This is the simplest solution where in fact all nodes transmit in each time slot with a fixed probability $\tau$. This probability is the inverse of the total nodes inside the network, that means $\tau=\frac{1}{N}$, where $N$ is the total number of active nodes inside the network, (nodes that compose the network).

We modeled the transmission of control packets by a one-dimensional transitory Markov chain, where each state represents the number of nodes attempting to transmit. As such, the chain starts at state $N$ and the final state (absorbing state) is 0 which corresponds to the case when all the nodes have successfully transmitted their control packets. In each state, the system can reduce the number of nodes attempting to transmit if a successful transmission occurs or it can remain in the same state if either a collision or an empty slot occurs.

Thus, the valid states of this model are as follows: $0<k \leq N$, where $k$ is the current number of nodes attempting to transmit. Notice that $k$ is a decreasing variable since once a node sends its control packet successfully, it does not try further transmissions. Therefore, we modeled the system as a stochastic process where nodes transmit their control packets successfully with probability $P_{s}$ and with probability $P_{f}$, the nodes do not transmit a packet in a particular time slot.

To compute $P_{s}$, we noticed that this is the probability of fulfill the following sentence: At state $k$ a successful transmission occurs when only one of the remaining $k$ nodes transmits with probability $\tau$. Hence, this can be described by a binomial random variable as follows:

$$
P_{s}(k)=\left(\begin{array}{l}
k \\
1
\end{array}\right) \tau(1-\tau)^{k-1}=k \tau(1-\tau)^{k-1}
$$

On the other hand, a non-successful transmission occurs if:

- None node of the remaining k nodes attempts to transmit a control packet, or,

- Two or more nodes try to transmit in the same time slot causing a collision in the channel. We assume that the transmission of at least two nodes is powerful enough to be detected in the system. 
Observe that these events are the complement of a successful transmission case. Hence, we can write the failure probability as:

$$
P_{f}(k)=1-P_{s}(k)
$$

Figure 1 shows the Markov chain that models this scheme, where with probability $P_{s}(k)$, it goes to a new state by a successful transmission and with probability $P_{f}(k)$ the chain remains in the same state $k$.

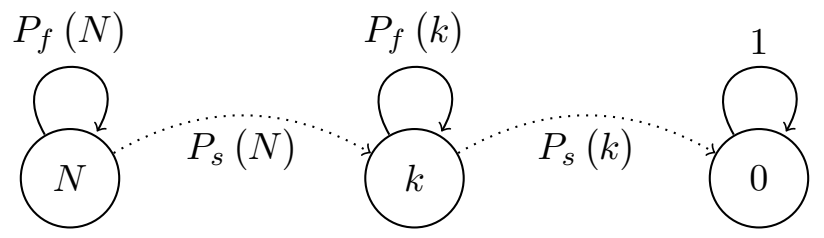

Fig. 1. Markov chain for control packets sending with fixed transmission probability

Although this strategy is simple, it has the disadvantage that it does not use an optimal transmission probability for the whole cluster formation phase since the number of nodes attempting to transmit is not constant along the cluster formation phase. At the beginning of this phase, this strategy reduces the collisions since it is more probable that only one of the $N$ nodes tries to transmit, but at the cost of many empty slots at the end of the cluster formation phase because the number of nodes attempting to transmit is, by much, lower than in first slots and the probability of transmission does not change by the time. To solve this problem, we present two adaptive schemes in the following subsections.

\subsection{Adaptive by Estimation Scheme (AES)}

In view the number of nodes attempting to transmit is not the same along the cluster formation phase, we propose an adaptive scheme. Note that at the beginning of the cluster formation phase, when all nodes in the system attempt to transmit their control packet, the transmission probability should be low in order to reduce the number of collisions in the shared channel. Conversely, when most of the nodes have successfully transmitted their control packet, the transmission probability should be high in order to avoid empty slots, taking advantage of the fact that packet collisions are uncommon.

As it is desirable that only one node transmits in each time slot, intuitively, the ideal transmission probability is inversely proportional to the amount of nodes attempting to transmit, then we denote this as $\tau_{a e}=\frac{1}{k^{\prime}}$, where $k^{\prime}$ is the number of nodes with pending transmission. Since in many cases, the actual number of remaining nodes cannot be known due to interference, noise or shadowing, we propose the use of an estimation value. Thus, in this scheme, the value of $\tau$ is updated in each time slot such that as the estimated number of nodes attempting to transmit decreases, the value of $\tau_{a e}$ increases. This implies that when a successful transmission is detected, the system updates the value of $\tau_{a e}$ 
by reducing its value proportionally. Thus the value of $\tau_{a e}$ for future slots is computed with the new estimation of $k^{\prime}$ until it occurs a new successful transmission and the value updates again.

Notice that now the value of nodes attempting to transmit has to be considered since it directly impacts to the transmission probability of the nodes to the sending of control packets. Although we are not using a specific method to estimate the number of nodes pending to transmit, it is possible since nodes sense the channel along the cluster formation phase, then they detect transmissions and estimate how many of them are pending to transmit. To this, it is only necessary that nodes know the value of $N$ or detect the number of transmissions in previous rounds.

Based on those considerations, we developed a new model to describe the transmission evolution of control packets of the nodes using this proposal of $\tau$ adaptive.

Again, we modeled the transmission of the nodes in the network through a Markov chain, but in this case, we chose a two-dimensional chain such that each state reflects both the actual number of nodes attempting to transmit $k$, and the estimated number of nodes attempting to transmit $k^{\prime}$, it is to say, the state is $\left(k, k^{\prime}\right)$. Notice that in an ideal channel without noise, interference, and shadowing $k=k^{\prime}$ since all the intended receivers successfully detect every transmission. However, in a more general case, where some transmissions are not received, we have the case where $k \neq k^{\prime}$. This chain starts in the state $(N, N)$ and finishes in the absorbing state $\left(0, k^{\prime}\right)$, which represents the case where all nodes have successfully transmitted their control packet, even if the estimated number of nodes is $k^{\prime} \neq 0$. As we are considering a free-error channel, the absorbing state 0,0 represents the finish of the sending of control packets.

For this new model, we have the same two main events: successful transmission, and the no successful transmission.

- Successful transmission: This happens when only one of the $k$ active nodes transmits. However, to compute the transmission probability, we use the estimated number of nodes in the system and the actual number of nodes pending to transmit. As such, this probability is:

$$
P_{s}\left(k, k^{\prime}\right)=\left(\frac{k}{k^{\prime}}\right)\left[1-\left(\frac{1}{k^{\prime}}\right)\right]^{k-1}
$$

Note that this probability is computed as (1) since the ratio $\frac{1}{k^{\prime}}$ is the transmission probability $\tau$ but in this case is updated when a successful transmission occurs.

- No successful transmission: This event presents when there are no transmissions in the slot, or when two or more nodes transmit in the same time slot causing a collision in the channel. The probability of this even occurs is:

$$
P_{f}\left(k, k^{\prime}\right)=1-P_{s}\left(k, k^{\prime}\right)
$$

In Figure 2 we show the aforementioned Markov chain. 


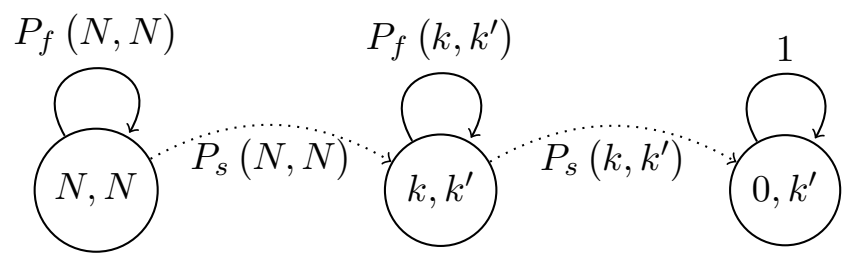

Fig. 2. Markov chain for the adaptive by estimation transmission probability scheme.

\subsection{Adaptive by Gamma Scheme (AGS)}

Another alternative for the selection of the transmission probability that we proposed before is the Adaptive by Gamma Scheme which aims at selecting the value of the transmission probability based on the outcome of the current slot. For this scheme, also the use of threshold values has to be carefully selected to provide QoS guarantees regarding system lifetime and reporting delay.

Especially, in the AGS scheme, nodes adjust their transmission probability in the following manner:

- If an empty slot occurs (no nodes transmit in such slot), the transmission probability is increased to prevent this from happening in the following slot. This event occurs with probability $P_{i}$ :

$$
P_{e}(k)=\left(1-\tau_{a g}\right)^{k}
$$

- If a slot is successful (a single transmission occurs in the current time slot), the transmission probability remains unchanged. This event occurs with probability $P_{c}$ :

$$
P_{s}(k)=k \tau_{a g}\left(1-\tau_{a g}\right)^{k-1}
$$

- If a collision occurs (two or more nodes transmit at the same time), the transmission probability is decreased to limit transmissions in the following slot. This event occurs with probability $P_{c}$ :

$$
P_{c}(k)=1-P_{s}-P_{i}
$$

We modeled this scheme with a two dimensional transitory Markov chain with the number of nodes attempting to transmit $k$ and the current value of $\tau_{a g}$. At the beginning of the cluster formation phase, $k=N$ and $\tau_{a g}=\frac{1}{N}$. Each state is represented by $k, \tau_{a g}$ and the chain evolves to new states where $k$ can remain unchanged or reduce, and the transmission probability is updated by a factor of $\gamma$, which increases or decreases at time $t$ according to the following cases:

- Successful transmission: We assume that the value of the transmission probability is adequate and it remains unchanged. As such, the update is:

$$
\tau_{a g}(t)=\tau_{a g}(t-1)
$$


- Empty slot: The transmission probability slightly increase by the same factor $\gamma$ as:

$$
\tau_{a g}(t)=\frac{1}{\gamma} \tau_{a g}(t-1)
$$

- Collision: The transmission probability is updated by a soft reduction by a factor of $\gamma$, thus the new value of $\tau_{a g}$ is computed as follows:

$$
\tau_{a g}(t)=\gamma \tau_{a g}(t-1)
$$

Where $0<\gamma<1$ and $t$ corresponds to the slot which presents the event.

In Figure 3 we show the chain transitions at state $\left(k, \tau_{a g}\right), 0 \leq k \leq N$.

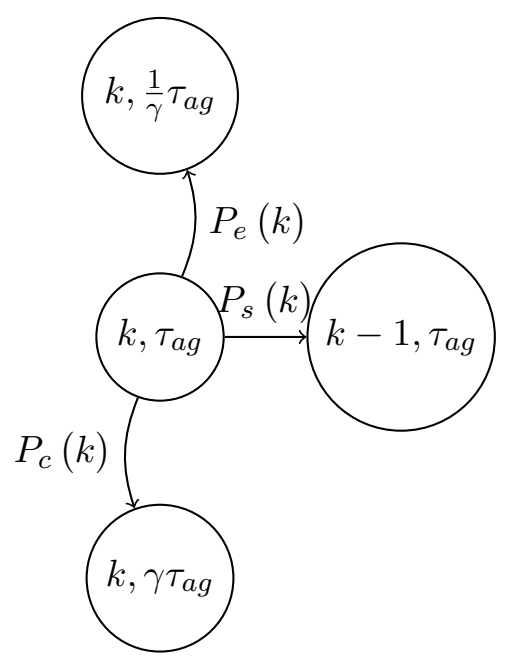

Fig. 3. Arbitrary state of the Markov chain for the adaptive by Gamma scheme, where $P_{e}(k), P_{c}(k)$, and $P_{s}(k)$ are the empty slot, collision, and successful transmission probabilities respectively.

\section{THRESHOLDS SELECTION}

In the previous section, we presented two adaptive schemes for the transmission of control packets in the cluster formation phase. Both schemes require a limit on the transmission probability in order to guarantee QoS metrics due to when $\tau$ is close to 1 or close to 0 , the performance of the system is considerably affected. In the case of AES we established one threshold: $\tau_{t h}$. In the case of AGS two thresholds are proposed: $\tau_{\max }$, and $\tau_{\min }$ and one control variable: $\gamma$. These thresholds directly impact the performance in energy consumption and delay by the cluster formation.

For example, in the scheme AES, for a system with $N=50$ and $\tau_{t h}=0.02$, the cluster formation delay and the energy consumption are 300 slots and $8 \times 10^{-4} J$ respectively, meanwhile for the same system with $N=50$ but with $\tau_{t h}=0.1$ the cluster formation 


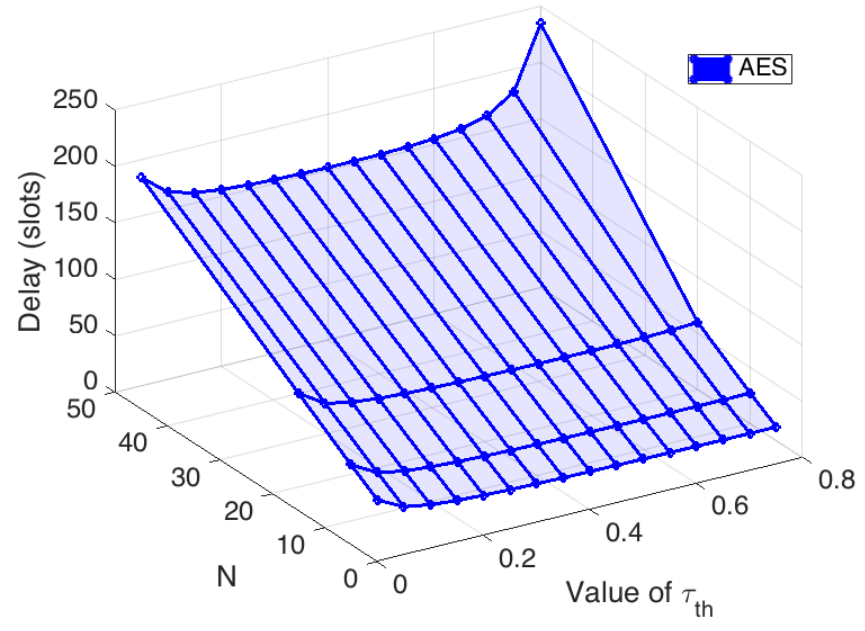

Fig. 4. Effect in the delay by the value of $\tau_{t h}$.

delay and the energy consumption decay to 189 slots and $4.8 \times 10^{-4} J$ respectively. The AGS is more sensitive for the threshold values. For example, if we change the value of $\gamma$ for a system with $N=50$, it is observable that for $\gamma=0.1$ the cluster formation delay and the energy consumption are 226 slots and $5.7 \times 10^{-4} \mathrm{~J}$ respectively meanwhile with $\gamma=0.95$ we have 144 slots and $4 x 10^{-4} J$ respectively.

Figure 4 and Figure 5 show this behavior, the first one presents the delay in AES for different values of $\tau_{t h}$ and the second one shows the delay for different values of $\gamma$ in AGS.

These figures and numerical results show that the thresholds need to be carefully selected to offer QoS guarantees. For instance in AES, consider the case with 50 nodes in the system, the minimum reporting delay is 150 slot approximately. As such, if the particular application requires a cluster formation time of fewer than 200 slots, a threshold value lower than 0.8 has to be selected. Otherwise, QoS in terms of average reporting delay cannot be met.

Moreover, for instance, in AGS, newly consider the case of 50 nodes in the system, the minimum reporting delay is 152 slot, approximately and this increases as the thresholds vary. As such, if the particular application requires a cluster formation time of this 152 slots, the threshold values of 0.02 and higher than 0.5 for $\tau_{\min }$ and $\tau_{\max }$ respectively has to be selected. Now we describe this issue in detail for each adaptive scheme.

\subsection{Thresholds of AES}

Although this adaptive strategy aims at nodes performing a single transmission per time slot, the main issue arrives at the end of the cluster formation phase. Specifically, 


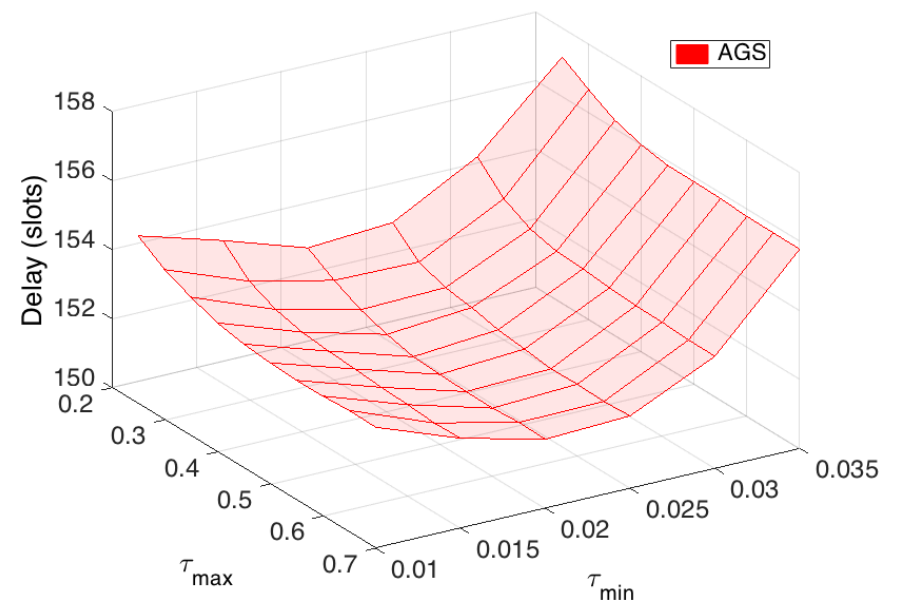

Fig. 5. Effect in the delay by the value of $\tau_{\min }$ and $\tau_{\max }$.

for two remaining nodes attempting to transmit, the transmission probability is set at $\tau_{e a}=\frac{1}{2}$ which causes many collisions. As such, it cannot guarantee QoS regarding energy consumption (system lifetime) and reporting delay (cluster formation delay). As such, to guarantee QoS, we propose to use a threshold such that it limits the value of $\tau_{e a}$ and reduces the access to the channel to avoid collisions. However, the value of threshold cannot be the same for networks composed of a different number of nodes. Therefore, the value of $\tau_{a e}$ is now computed as is described in the following equation:

$$
\tau_{a e}= \begin{cases}\frac{1}{k} & \text { if } k>n_{t h} \\ \tau_{t h} & \text { if } k \leq n_{t h}\end{cases}
$$

Where $n_{t h}$ corresponds to the threshold number of nodes after which the transmission probability does not decrease. This effectively posses a superior limit on the value of $\tau_{a e}$. Then, the probability of a successful transmission is computed according to the number of nodes estimated attempting to transmit and the threshold values are used to assure QoS guarantees. Therefore $P_{s}\left(k, k^{\prime}\right)$ is computed by the following equation:

$$
P_{s}\left(k, k^{\prime}\right)= \begin{cases}\left(\frac{k}{k^{\prime}}\right)\left[1-\left(\frac{1}{k^{\prime}}\right)\right]^{k-1} & \text { if } k>n_{t h} \\ k \tau_{t h}\left(1-\tau_{t h}\right)^{k-1} & \text { if } k \leq n_{t h}\end{cases}
$$

Notice that this threshold does not change the mathematical model for this scheme and, it only establishes a rule of how the transmission probability is updated. 


\subsection{Thresholds of AGS}

In this scheme, the need to carefully select the values of transmission probability threshold is relevant to provide QoS guarantees. The reason for this is as follows: First, in the case of continuous collisions, due to the random nature of the communication protocol, the value of $\tau_{a g}$ is decreased such that $\tau_{a g} \approx 0$. In this case, the channel access defines for many nodes in the network. Although this scheme effectively reduces collisions due to its adaptive nature, it may not provide QoS guarantees. To this end, we propose a minimum value, $\tau_{m i n}$, for the transmission probability. In the case of an empty slot, its value is increased by the $\gamma$ factor. On the other hand, if several idle slots occur, again due to the random nature of the protocol, the value of $\tau_{a g}$ is rapidly increased. This causes that $\tau_{a g} \approx 1$, causing many collisions that hinder the system operation. Hence, a maximum transmission probability threshold, $\tau_{\max }$, is introduced to provide QoS guarantees. Building the transmission probability is calculated as follows:

$$
\tau_{a g}(t)= \begin{cases}\left(\frac{1}{\gamma}\right) \tau_{a g}(t-1) & \text { if a idle slot is presented } \\ \gamma \tau_{a g}(t-1) & \text { if a collision is presented } \\ \tau_{\max } & \text { if }\left(\frac{1}{\gamma}\right) \tau_{a g}(t-1) \geq \tau_{\max } \\ \tau_{\min } & \text { if } \gamma \tau_{a g}(t-1) \leq \tau_{\min }\end{cases}
$$

Similarly, these thresholds do not change the analytical model and only impacts the system performance for the updating of the transmission probability. In the following section we present the results for the optimal thresholds computed experimentally for this study.

\section{NUMERICAL RESULTS}

In this section, we evaluate the performance of the system in terms of average energy consumption and average reporting delay for different system conditions. Additionally, we provide clear guidelines for the selection of the transmission probability thresholds in order to provide QoS guarantees in a cluster-based WSN.

First, we focus on the Adaptive by Estimation Scheme. Figure 6 shows the optimal values of $n_{t h}$, i.e., the values of $n_{t h}$ that entails the lowest energy consumption and cluster formation delay. Recall that nodes cannot report data until clusters are properly formed. Hence average cluster delay is also related to average reporting time. It can see that as the number of nodes in the system increases, the value of the threshold increases. This is because when $N$ is high, collision probability is also high and transmissions have to be further restricted. Based on this results, we provide a closed expression to calculate the optimal value of this threshold based on the number of active nodes $N$ as 


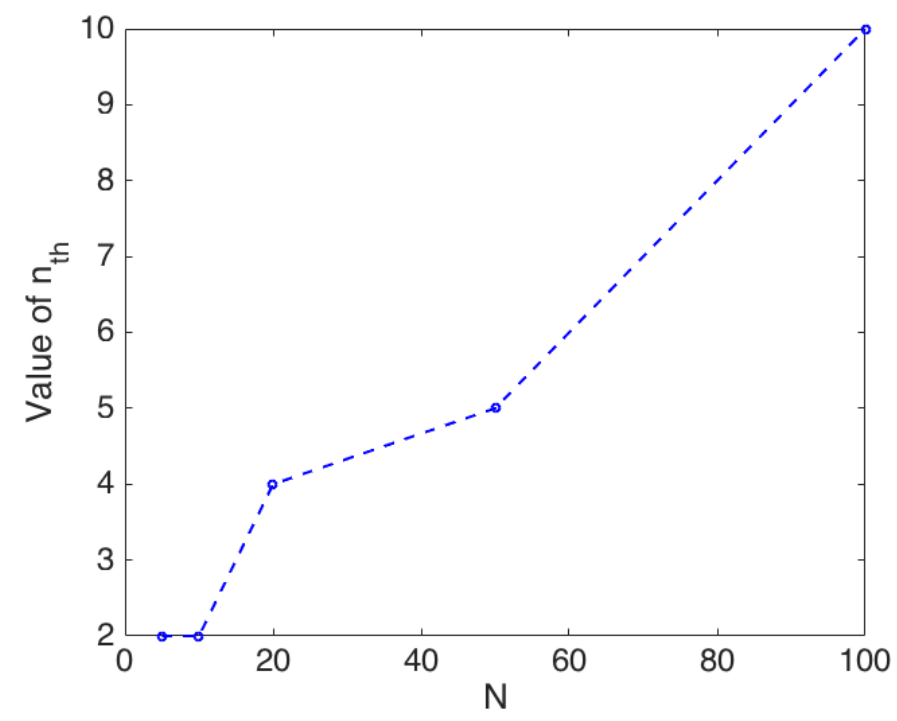

Fig. 6. Threshold of number of nodes attempting to transmit.

$n_{\text {th }}=$ round $\left(0.0002 N^{2}+0.62 N+1.8\right)$. Figure 7 presents results for the optimal values of the transmission probabilities for a different number of nodes. Again, this threshold can be selected according to the following approximate expression: $\tau_{t h}=0.0001 N^{2}-0.017 N+0.74$. we calculated these expressions by a linear regression procedure where the solution for the equations system that better adjust to a polynomial of second degree according to the values of $N$ and the results obtained in the experimental analysis.

Now, we study the Adaptive by Gamma Scheme. Figure 8 shows the optimum values for the two thresholds for the transmission probabilities (lower and upper) and the optimum value for $\gamma$. It can see that the lower and upper bounds are a decreasing function of $N$. On the other hand, the higher the value of $N$ the higher the optimum value of $\gamma$. The reason for this behavior is that, when $N$ is high, lower transmission probabilities reduce collisions. Conversely, for low values of $N$, there is lower collision probability, and the transmission probability can increase as seen by the higher thresholds. Another significant result is that the adjustment on the transmission probability has to be higher for high values of $N$ in order to achieve better performance. We have also found closed expressions to calculate these thresholds and the value of $\gamma$ for a practical network as follows: $\tau_{\min }=0.0001 N^{2}-0.0072 N+0.22$ and $\tau_{\max }=0.85-0.0045 N$ and $\gamma=0.46 N+0.7$.

Now, the optimal performance, i.e., the lowest average energy consumption and reporting delay achieved by selecting the optimal values of the lower and upper thresholds and $\gamma$, are presented in Figure 9 and Figure 10 respectively. We can see that the adaptive schemes improve over $40 \%$ of the energy consumption compared to the fixed scheme in systems 
14 - E. Romo-Montiel et al.

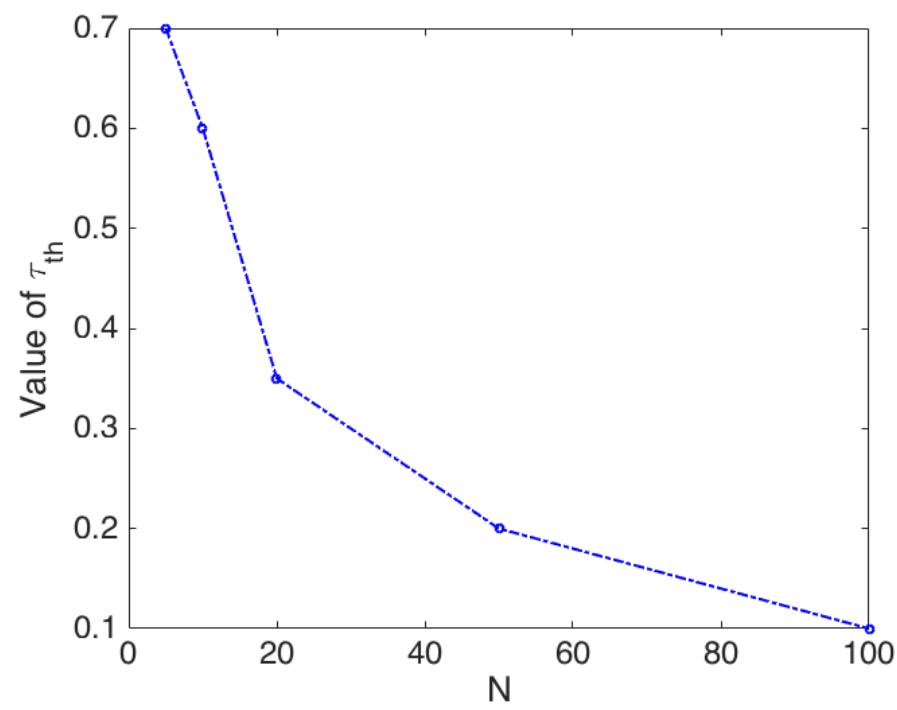

Fig. 7. Value of $\tau_{t h}$.

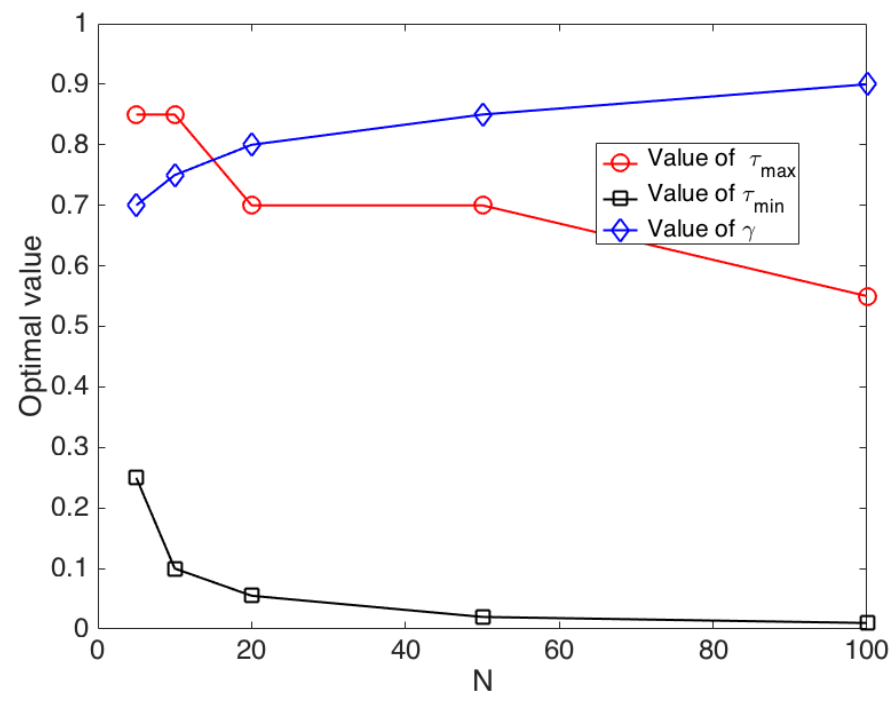

Fig. 8. Optimal thresholds for AGS.

with $N>20$ and the same reduction for the average cluster formation delay for $N>10$. Moreover, the AGS has better performance than the AES strategy for networks with $N>20$. Additionally, to provide QoS, the AGS scheme has the advantage of allowing a fine tuning of the system performance by carefully selecting the value of $\gamma$ in combination 
with both upper and lower thresholds, while the AES scheme only allows for the selection of $\tau_{t h}$.

Although the incremental network lifetime is not explicitly given, clearly we can deduce that as the energy consumption is reduced during the cluster formation process the network lifetime increases. This work proves that the correct use of thresholds for the transmission probability in systems with contending processes increases the system performance since it reduces the energy consumption and packet delay.

Additionally, we are not using a standard metric unit in the delay results. This is because we are studying the impact of the use of optimal thresholds and such metric can be found readily scaling to the size of the slot that the users prefer. For example, if we adopt the packet size of 2000 bits used by Heinzelman in [11] and a transmission rate of $24 \mathrm{Mbps}$ the slot size is about $83 \mu \mathrm{s}$ while for a packet of 500 bits the slot size is about $20 \mu \mathrm{s}$.

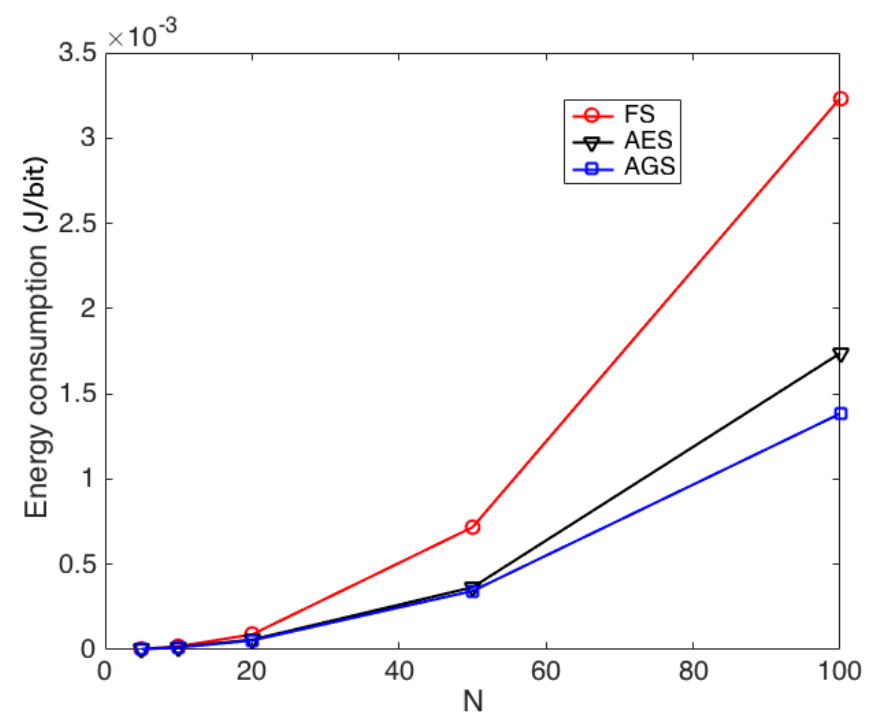

Fig. 9. Energy consumption in control packets sending process

\section{CONCLUSION}

In this paper we study, analyze and evaluate the impact of the transmission probability thresholds in adaptive schemes for QoS provision in WSNs. In the literature, the adaptive schemes have been extensively studied, but the threshold values have been largely overlooked. Numerical results show that a careful selection of the threshold values has to be made in order to assure QoS levels that cannot be assured by the use of adaptive schemes alone. Furthermore, the AGS strategy achieves lower average energy consumption (higher lifetime) and average cluster formation delay (reporting delay) than the AES scheme, with 


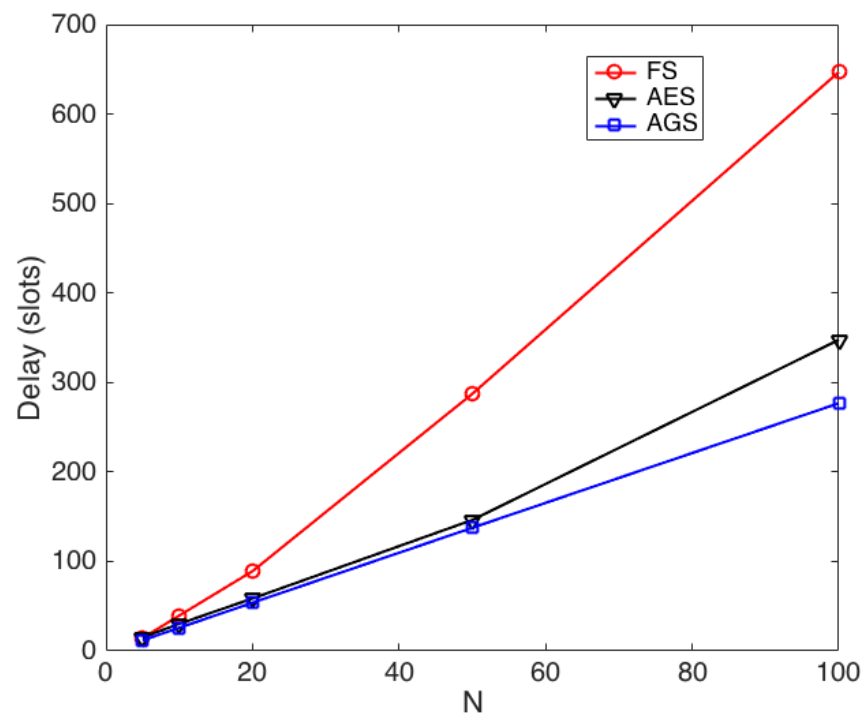

Fig. 10. Delay in control packets sending process

the additional advantage of allowing a finer control on the system performance through the value of $\gamma$. Finally, we have provided closed expressions for the optimum transmission probability values to use in a practical system for future work.

\section{ACKNOWLEDGMENTS}

The authors would like to thank to the Instituto Politécnico Nacional, and CONACYT for providing the facilities to develop this work.

The work is supported by Sección de Investigación y Posgrado in special by the project No. SIP 20181929 named Diseño e implementacion de redes neuronales de reservaria con base en FPGAs para claisficación de datos.

\section{REFERENCES}

[1] December 1975. Packet Switching in Radio Channels: Part I - Carrier Sense Multiple-Access Modes and Their Throughput-Delay Characteristics. IEEE Transactions on Communications vol. 23, No. 12 (December 1975), p.p. 1400-1416. https://doi.org/10.1109/TCOM.1975.1092768

[2] June 2015. LEACH and its Improved Versions-A Survey. International Journal of Scientific \& Engineering Research vol. 6, 6 (June 2015), p.p. 184-188.

[3] M. Mehdi Afsar and Mohammad-H. Tayarani-N. 2014. Clustering in sensor networks: A literature survey. Journal of Network and Computer Applications 46 (2014), 198 - 226. https://doi.org/10. 1016/j.jnca.2014.09.005

[4] Paolo Baronti, Prashant Pillai, Vince W.C. Chook, Stefano Chessa, Alberto Gotta, and Y. Fun Hu. 2007. Wireless sensor networks: A survey on the state of the art and the 802.15.4 and ZigBee standards. 
Computer Communications 30, 7 (2007), 1655 - 1695. https://doi.org/10.1016/j.comcom.2006.12.020 Wired/Wireless Internet Communications.

[5] L. M. Bello, P. Mitchell, and D. Grace. 2014. Application of Q-Learning for RACH Access to Support M2M Traffic over a Cellular Network. In European Wireless 2014; 20th European Wireless Conference.

[6] Madhav Bokare and Mrs Anagha Ralegaonkar. 2012. Wireless sensor network: A promising approach for distributed sensing tasks. Excel Journal of Engineering Technology and Management Science 1, 1 (2012), 1-9.

[7] V. Cionca, T. Newe, and V. Dadârlat. 2008. TDMA Protocol Requirements for Wireless Sensor Networks. In 2008 Second International Conference on Sensor Technologies and Applications (sensorcomm 2008). 30-35. https://doi.org/10.1109/SENSORCOMM.2008.69

[8] G. del Angel and T. L. Fine. 2004. Optimal power and retransmission control policies for random access systems. IEEE/ACM Transactions on Networking 12, 6 (Dec 2004), 1156-1166. https: //doi.org/10.1109/TNET.2004.838605

[9] Hossam Mahmoud Ahmad Fahmy. 2016. Wireless Sensor Networks, Concepts, Applications, Experimentation and Analysis. http://www.springer.com/gp/book/9789811004117

[10] Fakhrosadat Fanian, Marjan Kuchaki Rafsanjani, and Vahid Khatibi Bardsiri. 2016. A Survey of Advanced LEACH-based Protocols. International Journal of Energy, Information and Communications Vol.7, 1 (2016), p.p.1-16. https://doi.org/10.14257/ijeic.2016.7.1.01

[11] W.R. Heinzelman, A. Chandrakasan, and H. Balakrishnan. 2000. Energy-efficient communication protocol for wireless microsensor networks. In System Sciences, 2000. Proceedings of the 33rd Annual Hawaii International Conference on. 10 pp. vol.2-. https://doi.org/10.1109/HICSS.2000.926982

[12] M. S. Islam, M. Hoq, M. A. S. Haque, M. A. R. Akand, M. R. Hasan, and M. K. Basher. 2014. Challenges and prospects of cost-effective Si-based solar cells fabrication in Bangladesh. In Electrical Engineering and Information Communication Technology (ICEEICT), 2014 International Conference on. 1-6. https://doi.org/10.1109/ICEEICT.2014.6919168

[13] A. Mendez and D. Covarrubias. 2001. Stability and optimal retransmission control of S-Aloha as a RACH channel on wireless networks. In IEEE 54th Vehicular Technology Conference. VTC Fall 2001. Proceedings (Cat. No.01CH37211), Vol. 3. 1368-1372 vol.3. https://doi.org/10.1109/VTC. 2001.956420

[14] M. S. Mohd Resali and H. Salleh. 2010. Comparison of energy harvesting power management techniques and application. In Electronic Manufacturing Technology Symposium (IEMT), 2010 34th IEEE/CPMT International. 1-5. https://doi.org/10.1109/IEMT.2010.5746768

[15] Edgar Romo-Montiel, Mario E. Rivero-Angeles, Gerardo Rubino, Heron Molina-Lozano, Rolando Menchaca-Mendez, and Ricardo Menchaca-Mendez. 2017. Performance Analysis of Cluster Formation in Wireless Sensor Networks. Sensors 17, 2902 (2017). http://www.mdpi.com/1424-8220/17/12/2902

[16] W. Xuegang, Z. Xiaoping, F. Bin, Y. Liu, and Z. Wei. 2017. An energy-balance and game-theorybased cluster formation method for wireless sensor networks. International Journal of Distributed Sensor Networks 13 (2017). https://doi.org/10.1177/1550147717720792 